Anais da Academia Brasileira de Ciências (2002) 74(1): 33-35

(Annals of the Brazilian Academy of Sciences)

ISSN 0001-3765

www.scielo.br/aabc

\title{
Surfaces of Constant Mean Curvature in Euclidean 3-space Orthogonal to a Plane along its Boundary
}

\author{
PEDRO A. HINOJOSA \\ Centro de Ciências Exatas e da Natureza, Departamento de Matemática \\ Universidade Federal da Paraíba, João Pessoa, Brasil, and \\ Universidade Federal do Ceará, Centro de Ciências, Pós Graduação em Matemática \\ Campus do Pici, Bloco 914 - 60455-760 Fortaleza, Ce, Brasil \\ Manuscript received on October 25, 2001; accepted for publication on November 28, 2001; \\ presented by J. LuCAS BARBOSA

\begin{abstract}
We consider compact surfaces with constant nonzero mean curvature whose boundary is a convex planar Jordan curve. We prove that if such a surface is orthogonal to the plane of the boundary, then it is a hemisphere.
\end{abstract}

Key words: surfaces with boundary, constant mean curvature, elliptic partial differential equation.

Let $\mathrm{M}$ be a compact surface inmersed in $\mathbb{R}^{3}$ with constant mean curvature $H$ whose boundary $\partial M=\Gamma$ is a planar Jordan curve of length $L$. Let $D$ be a planar region enclosed by $\Gamma$ and let $A$ be the area of $D$. Let us consider the cycle $M \cup D$ oriented in such a way that its orientation, along $M$, coincides with the one defined by the mean curvature vector. Let $Y$ be a Killing vector field in $\mathbb{R}^{3}$ and $n_{D}$ be a unitary vector field normal to $D$ in the orientation of $M \cup D$. Let $v$ be the unitary co-normal vector field along $\partial M=\Gamma$ pointing inwards $M$. By the flux formula it is known that $|H| \leq \frac{L}{2 A}$ where equality holds if and only if $v=n_{D}$. That is, if and only if $v$ is constant and orthogonal to $D$ along $\Gamma$.

In this work we consider the case $|H| \leq \frac{L}{2 A}$ and we show that, in the above conditions, if $M$ is embedded and $\Gamma$ is convex, then $M$ is a hemisphere. Explicitly we prove that:

THEOREM 1. Let $M$ be a compact embedded surface in $\mathbb{R}^{3}$ with constant mean curvature $H \neq 0$ whose boundary $\partial M$ is a Jordan curve $\Gamma$ in a plane $\mathbb{P} \subset \mathbb{R}^{3}$. Suposse that $\Gamma$ is convex and $M$ is perpendicular to the plane $\mathbb{P}$ along its boundary. Then $M$ is a hemisphere of radius $\frac{1}{|H|}$.

Correspondence to: Universidade Federal do Ceará

e-mail: hinojosa@mat.ufpb.br 
This theorem generalizes a result obtained by Brito and Earp (Brito and Earp 1991). We succed in discarding their assumption that $\partial M$ should be a circle of radius $\frac{1}{|H|}$.

A sketch of the proof of the theorem is as follows.

First, under the hypothesis of the theorem, $M$ must be totally contained in one of the halfplanes determined by $\mathbb{P}$ (see (Brito et al. 1991), for example). Now let $M^{*}$ be the reflection of $M$ with respect to the plane $\mathbb{P}$. Since $M$ is orthogonal to $\mathbb{P}$ along $\Gamma$, we have that $\tilde{M}:=M \cup M^{*}$ is a compact surface without boundary, embedded in $\mathbb{R}^{3}$. Note that a priori $\tilde{M}$ is only of class $C^{1}$ along $\Gamma$. We will prove that $\tilde{M}$ is at least of class $C^{3}$. In this way we are able to use a classical result due to Alexandrov (see (Hopf 1983), for example) in order to establish that $\tilde{M}$ is a sphere and therefore $M$ is a hemisphere.

The regularity of $\tilde{M}$ along $\Gamma$ is achieved by means of the theory of elliptic partial differential equations. Let $p$ be any point in $\Gamma \subset \widetilde{M}$ and $\Omega$ be an open neighborhood of 0 in $T_{p} \tilde{M}$ chosen in such a way that locally around $p, \tilde{M}$ may be described as the graph of a function $u: \Omega \rightarrow \mathbb{R}$. For our purposes, it is suffices to consider $\Omega$ of class $C^{1,1}$.

It is clear that $u \in C^{1}(\Omega)$. So, $\nabla u$ is well-defined and continuous. Since $\Omega$ is bounded we have that $u \in W^{1,2}(\Omega)$.

Let us denote the linear space of k-times weakly differentiable functions by $W^{k}(\Omega)$. For $p \geq 1$ and $\mathrm{k}$ a non-negative integer, we let $W^{k, p}(\Omega)=\left\{u \in W^{k}(\Omega), D^{\sigma} u \in L^{p}(\Omega)\right.$ for all $\left.|\sigma| \leq k\right\}$.

The Hölder spaces $C^{k, \alpha}(\Omega)$ are defined as the subspaces of $C^{k}(\Omega)$ consisting of functions whose k-th order partial derivatives are locally Hölder continuous whith exponent $\alpha$ in $\Omega$.

We define on $\Omega$ the following linear operators:

$$
L_{1} v:=D_{i}\left(a^{i j} D_{j} v\right), \quad v \in W^{1,2}(\Omega) \quad i, j=1,2
$$

and

$$
L_{2} v:=A^{i j} D_{i j} v, \quad v \in W^{2,2}(\Omega) \quad i, j=1,2,
$$

where the coefficients $a^{i j}$ are given by

$$
a^{11}=a^{22}=\frac{1}{1+|\nabla u|^{2}}, \quad a^{12}=a^{21}=0
$$

and the coefficients $A^{i j}$ are defined by $A^{11}=1+u_{y}^{2}, A^{12}=A^{21}=-u_{x} u_{y}, A^{22}=1+u_{x}^{2}$. Finally, the symbols $D_{i}, D_{i j}, i, j=1,2$ stand for partial differentiation.

We prove that $u$ is a weak solution to the equation $L_{1} u=2 H$. By the Corollary 8.36 (Gilbarg and Trudinger 1983) we have $u \in C^{1, \alpha}(\Omega)$. Moreover, by the Lebesgue's dominated convergence theorem and Lemma 7.24 (Gilbarg and Trudinger 1983) we can conclude that $u \in W^{2, p}\left(\Omega^{\prime}\right)$ for any subdomain $\Omega^{\prime} \subset \subset \Omega$. Fixed $\Omega^{\prime} \subset \subset \Omega$, we consider the equation

$$
L_{2} v=2 H\left(1+|\nabla u|^{2}\right)^{\frac{3}{2}} .
$$


We observe that $u \in W^{2,2}\left(\Omega^{\prime}\right)$. Thus, $L_{2} u$ is well-defined. Moreover, we have that $L_{2} u=2 H$ $\left(1+|\nabla u|^{2}\right)^{\frac{3}{2}}$ in $\Omega^{\prime}$. It means that $u \in W^{2,2}\left(\Omega^{\prime}\right)$ is a solution to the equation (1) just above. Now using the Theorem 9.19 (Gilbarg and Trudinger 1983) we obtain $u \in C^{2, \alpha}\left(\Omega^{\prime}\right)$. Repeating the same procedure we conclude that $u \in C^{\infty}\left(\Omega^{\prime}\right)$. Thus, $\widetilde{M}$ is $C^{\infty}$. So, $\widetilde{M}$ is a regular compact closed surface embedded in $\mathbb{R}^{3}$ with constant mean curvature. By the Theorem 5.2 (Chapter V, (Hopf 1983)) we conclude that $\tilde{M}$ is a (round) sphere and therefore $M$ is a hemisphere.

\section{ACKNOWLEDGMENTS}

This work is part of my Doctoral Thesis at the Universidade Federal do Ceará - UFC. I want to thank my advisor, Professor J. Lucas Barbosa, and also Professor A. G. Colares, by the encouragement and many helpful conversations. The research was supported by a scholarship of CAPES.

\section{RESUMO}

Consideramos superfícies compactas com curvatura média constante e não nula as quais têm como bordo uma curva de Jordan plana convexa. Provamos que, se uma tal superfície é ortogonal ao plano do bordo então é um hemisfério.

Palavras-chave: superfícies com bordo, curvatura média constante, equações diferenciais parciais elípticas.

\section{REFERENCES}

Brito F AND EARP RS. 1991. Geometric Configurations of Constant Mean Curvature Surfaces with Planar Boundary. An Acad bras Ci 63: 5-19.

Brito F, Earp RS, Meeks W and Rosenberg H. 1991. Structure Theorems for Constant Mean Curvature Surfaces Bounded by a Planar Curve. Indiana Univ Math J 40(1): 333-343.

Gilbarg D and Trudinger NS. 1983. Elliptic Partial Differential Equations of Second Order. 2nd edition, Springer-Verlag, Berlin.

Hopf H. 1983. Differential Geometry in the Large. Lectures Notes in Mathematics, 1000, Springer-Verlag, Berlin. 